\title{
Funcionamiento familiar y severidad de los problemas asociados a la adicción a drogas en personas que solicitan tratamiento
}

\author{
Ioseba Iraurgi Castillo ${ }^{1}$, Mireia Sanz Vázouez ${ }^{2}$, Ana Martínez-Pampliega ${ }^{3}$ \\ 1.- Módulo de Asistencia Psicosocial de Rekalde. Bizkaia. \\ 2.- Servicio de Drogodependencias. Ayuntamiento de Derio. Bizkaia \\ 3.- Facultad de Psicología. FICE. Universidad de Deusto. Bizkaia.
}

Enviar correspondencia a:

Ioseba Iraurgi Castillo. Módulo de Asistencia Psicosocial de Rekalde. Camilo Villabaso 24 lonja. 48002 Bilbao. e-mail: iraurgi@euskalnet.net

Recibido: 6 de noviembre de 2003. Aceptado: 24 de abril de 2004.

\section{RESUMEN}

A través de un diseño observacional de corte transversal se evalúa la situación de 140 personas con diagnóstico por trastorno adictivo a sustancias no alcohólicas que inician tratamiento en 15 centros de tratamiento a toxicomanías (CTT) de la provincia de Bizkaia (España) y se explora el funcionamiento familiar y su relación con los problemas derivados o asociados a esta situación. La situación sociofamiliar se valorá a través del FAP (Family Assessment Package) y el grado de severidad de la adicción mediante el EuropASI por un evaluador entrenado ajeno a los CTT. Para el ánalisis de datos se han utilizado técnicas de correlación. Se ha observado una valoración diferencial de la severidad de la adicción entre el evaluador (VGE) y el evaluado (VGP). El primero tiende a primar sobre el segundo la gravedad del consumo de sustancias (VGE = 10 vs $V G P=7,20 ; p<0,01$ ), mientras el segundo prima sobre el primero la severidad de la situación sociofamiliar (VGP=5,62 vs $V G E=4,74 ; p<0,01)$ y psiquiátrica $(V G P=5,02$ vs $V G E=4,06 ; p<0,01)$. Por otra parte, se observa como una peor situación médica y psiquiátrica se asocia positivamente con un mayor estrés familiar $(r=0,22$ y $r=$ $0,27$ respectivamente, $p<0,01)$, y el uso de drogas se asocia con un mayor estrés familiar $(r=0,26 ; p<0,01)$, con una más baja satisfacción familiar $(r=-0,26 ; p<0,01)$, y con una peor comunicación y recursos familiares $(r=-0,18$ y $r=-0,21$ respectivamente; $p<0,05)$. Por tanto, se constata la relación entre la severidad de la adicción y el funcionamiento familiar, tanto desde la apreciación del clínico como desde la del propio paciente.

Palabras clave: Funcionamiento familiar, Severidad de la adicción, Tratamiento, Drogodependencia, EuropASI, FAP.

\section{ABSTRACT}

Using a cross-section observational design, we examined the situation of 140 people diagnosed with an addiction to non-alcoholic substances who initiated treatment in 15 drug treatment centres in Bizkaia (Spain), and explored family functioning and its relationship with the problems derived from or associated with this situation. The socio-family situation was evaluated through the Family Assessment package (FAP) and the degree of severity of addiction through the EuropASI by an externally and independently trained assessor. Correlation techniques were used in the data analyses. Differences were found in the evaluation of the severity of the addiction by the assessor $(A)$ and the patient $(P)$. The assessor perceives a higher severity of substance use $(A=10 \mathrm{v} . P=7.20 ; p<0.01)$, and the patient perceives a higher severity in the social and family situation $(P=5.62 \mathrm{v} . A=4.74 ; p<0.01)$ and the psychiatric situation $(P=5.02$ v. $A=4.06 ; p<0.01)$ in comparison with the assessor. In addition, the results show how a worse medical and psychiatric situation is associated with higher family stress $(r=0.22$ and $r=0.27$ respectively, $p<0.01)$, and drug use is associated with higher family stress $(r=0.26 ; p<0.01)$, lower family satisfaction $(r=-0.26 ; p<0.01)$ and less communication and family resources $(r=-0.18$ and $r=-0.21$ respectively; $p<0.05)$. Therefore, the results show the relationship between addiction severity and family functioning, when assessed by both an assessor and a patient.

Key words: Family Functioning, Addiction Severity, Treatment, Drug Dependence, EuropASI, FAP.

\section{INTRODUCCIÓN}

$\mathbf{L}$ a familia ha sido siempre reconocida como una de las variables más relevantes en el origen y mantenimiento del consumo de drogas. La asociación entre el funcionamiento familiar y el consumo de estas sustancias por parte de los hijos ha sido objeto de numerosas investigaciones ${ }^{(1-6)}$. La mayor parte de estos estudios resaltan la necesidad de implicar a la familia en la prevención y tratamiento de las toxicomanías (6-7). 
Determinados estudios ${ }^{(8-10)}$ confirman la relación entre el uso de drogas en los hijos y un ambiente familiar conflictivo y hostil. De forma genérica, se sostiene que la crianza de niños de familias con alto nivel de conflicto es un factor de riesgo para el desarrollo de trastornos de conducta ${ }^{(11)}$

Algunos factores característicos de la estructuración familiar, tales como la comunicación, el nivel de satisfacción, los recursos de manejo en las relaciones familiares y el nivel de estrés, han mostrado evidencia de su impacto.

\section{Comunicación familiar.}

Muchos estudios confirman la importancia de la comunicación paterno-filial. Como señala Castells ${ }^{(12)}$, la familia debe buscar momentos de comunicación en los que exista un adecuado intercambio que sirva para orientar y encauzar al adolescente en la búsqueda de su identidad, al mismo tiempo que se le permita sentirse querido y apoyado. Cuando esto no existe y se da incomunicación entre padres e hijos, pueden existir consecuencias serias para el adolescente ${ }^{(13)}$. En referencia al consumo de drogas, numerosos autores destacan dificultades en la comunicación de las familias de drogodependientes ${ }^{(3-4,14-15)}$.

\section{Satisfacción familiar.}

Las relaciones de insatisfacción respecto al medio socializador familiar, han sido señaladas como destacado factor a tener en cuenta en el consumo de drogas en los adolescentes ${ }^{(16-19)}$. Vielva y colaboradores ${ }^{(20)}$ encuentran que existe una menor satisfacción con el funcionamiento familiar, tanto desde la percepción de los hijos como de los padres, en aquellas familias en las cuales los hijos consumen drogas, lo cual sugiere que la falta de satisfacción podría estar en la base del consumo.

\section{Recursos familiares}

Los recursos familiares son aquellos aspectos sociales, emocionales y físicos que hacen que la familia sea menos vulnerable y más capaz de tratar con factores estresantes, facilitando la adaptación individual y familiar (21-22). Las escasas investigaciones Ilevadas a cabo en relación a esta variable ${ }^{(23)}$ apoyan la hipótesis de que los jóvenes procedentes de familias con más recursos son menos susceptibles a tener problemas de abuso de drogas.

\section{Estrés familiar}

El consumo de drogas ha sido siempre asociado a eventos estresantes de la familia como desavenencias conyugales, disputas entre los padres, etc. (24-27). La mayor parte de las investigaciones soportan la hipótesis de Lewis ${ }^{(28)}$, quien identifica la calidad de la relación marital como uno de los factores más importantes asociados al nivel de competencia o de salud de la familia. Tampoco pasa inadvertido que la convivencia familiar cuando alguno de sus miembros adolece una enfermedad o es usuario de drogas, está marcada por un continuo y alto nivel de estrés ${ }^{(5,29)}$.

Estos factores, entre otros de ámbito familiar (30), se hallan en la etiología de los problemas de abuso de drogas, pero también constituyen factores implicados en el mantenimiento del problema una vez aparecido. Desde esta perspectiva, la hipótesis subyacente es que un desajuste en este tipo de factores familiares estarán claramente presentes entre personas que solicitan tratamiento por abuso o dependencia de drogas.

Nuestro objetivo, en el presente estudio, es explorar el funcionamiento familiar de personas que solicitan tratamiento por su toxicomanía y su relación con los problemas derivados o asociados a esta situación.

\section{MÉTODO}

\section{Diseño}

Para la satisfacer los objetivos planteados se recurrió a una estrategia correlacional, optándose por un diseño transversal.

\section{Muestra}

La muestra está constituida por 140 personas con diagnóstico de adicción a opiáceos ${ }^{(31)}$ que inician tratamiento en varios Centros de Asistencia a Toxicomanías (CAT) de la provincia de Bizkaia. Los sujetos fueron reclutados de un total de 15 CATs y dieron consentimiento de participación en el estudio.

El perfil del entrevistado fue el siguiente: hombre $(89,3 \%)$, de 28,6 años de edad (Desviación Estándar - $\mathrm{DE}=6,0)$, soltero $(80,7 \%)$, con bajo nivel educativo $(60,7 \%$ no han superado la EGB), en situación de paro $(59,3 \%)$, y que convive con su familia de origen $(77,1 \%)$. Respecto a la historia adictiva, se observa una evolución del consumo de 6,5 años ( $D E=5,3)$, con una media de tratamientos previos de 0,3 (DE= 
$1,0)$; en un $90,7 \%$ de los casos el tratamiento que iniciaban cuando fueron entrevistados era el primero y sólo un 4,3\% había realizado más de dos tratamientos previos. Un 68,6\% de los casos ha utilizado en alguna ocasión la vía inyectada, haciendo uso actual de esta vía el 47,9\%. La prevalencia de seropositividad a VIH es del $15 \%$, siendo para el caso de la hepatitis del $68,6 \%$.

\section{Procedimiento}

Un miembro del equipo de investigación contactó con cada uno de los CATs participantes y solicitó su colaboración en el estudio. Tras atender la demanda en cada caso, el clínico de referencia para cada sujeto le invitaba a participar en el estudio y en caso de aceptación propiciaba una cita con el investigador externo para la entrevista de evaluación. En esta entrevista individualizada se informaba al paciente de los objetivos del estudio y se volvía a solicitar su consentimiento de participación. La entrevista tenía una duración media de entre 60 y 90 minutos, en la cual se recogían datos de filiación y de historia de consumo de drogas, se administraba la entrevista clínica EuropASI y el FAP, cuestionario de 76 ítems autoadministrado para evaluar el sistema familiar (véase instrumentos). Todos los sujetos fueron entrevistados por la misma persona, ajena a los equipos clínicos de los CATs, con entrenamiento en el manejo de la entrevista clínica EuropASI.

\section{Instrumentos}

Dos instrumentos constituyen el núcleo del estudio: el FAP y el EuropASI.

FAP (Family Assessment Package) (32) / Batería de Evaluación Familiar.-

Desarrollada por Olson, Tiesel, Gorall y Fitterer en 1996, es una batería de cuestionarios autoaplicados que incluye cinco instrumentos de evaluación familiar independientes: el 'Family Stress Scale', el 'Family Communication Scale', el 'Family Satisfaction Scale', el 'Family Strengths Scale', y el 'FACES-IV'. Las características descriptivas y el estudio de adaptación cultural y validación psicométrica de este instrumento ya han sido expuestas en otro lugar ${ }^{(33)}$. De estos cinco instrumentos, los cuatro primeros mostraron una buena adaptación al castellano no así el FACES-IV, por lo que en el presente estudio se desestimó la utilización del mismo para la valoración del funcionamiento familiar. Describiremos a continuación las características de las escalas del FAP utilizadas, pudiéndose consultar las mismas en el anexo.

Family Stress Scale ${ }^{(34)}$ / Escala de Estrés Familiar.Se trata de una adaptación de 20 ítems del 'Family
Inventory of Life Events and Changes' de McCubbin, Patterson y Wilson ${ }^{(35)}$ que trata de evaluar la tensión en los ámbitos de vida familiar. En la versión inglesa presenta coeficientes de consistencia interna de 0,81 y fiabilidad test-retest entre 0,72 y 0,77. En el estudio de adaptación al castellano se han obtenido índices de 0,82 y 0,83, respectivamente, y una correlación intraclase $(\mathrm{CCl})$ de 0,87.

Family Communication Scale (36) / Escala de Comunicación Familiar.- Se trata de una versión corta (12 ítems) del 'Parents-Adolescent Communication' creado por los mismos autores. Trata de medir tanto aspectos positivos (libertad para el intercambio de información y emociones, comprensión, etc.) como negativos (dificultad para compartir, estilo negativo de interacción, etc.) de la comunicación. En su versión original mostró una adecuada fiabilidad que se ha reproducido en la versión en castellano; en ambos casos con valores alpha por encima de 0,88.

Family Satisfaction Scale (37) / Escala de Satisfacción Familiar.- Constituida por 10 ítems, trata de medir el grado de satisfacción experimentado con los aspectos relacionados con la cohesión y adaptabilidad familiar. Al igual que las escalas anteriores ha mostrado buenos criterios de fiabilidad tanto en la versión original (alpha de Cronbach $(\alpha)$ de 0,91 y coeficiente testretest de 0,75$)$ como en la adaptación española $(\alpha=$ $0,92$; test-retest $=0,94 ; \mathrm{CCl}=0,95)$.

Family Strengths Scale (38) / Escala de Recursos Familiares.- Formada por 12 ítems, evalúa la competencia, orgullo, lealtad, confianza, respeto y acuerdo entre los miembros de la familia. Esta escala también reúne características psicométricas muy adecuadas con valores de fiabilidad por encima de 0,83; en la adaptación española se ha encontrado un alpha de Cronbach de 0,85, valores test-retest de 0,88 y CCl de 0,91 .

Estas cuatro escalas mantienen una relación lineal con el funcionamiento familiar. Salvo la escala de estrés, todas las escalas presentan un sentido positivo, de forma que a mayor puntuación en cada una de estas dimensiones sería indicativo de un mejor funcionamiento familiar; en el caso del estrés, la relación ha de entenderse de forma inversa -a mayor puntuación peor funcionamiento familiar-. En todos los casos, y para permitir la comparación entre los valores alcanzados en cada escala, se procedió a una transformación decimal de sus puntuaciones de forma que éstas oscilen entre cero y diez.

EuropASI (European Addiction Severity Index / Índice de Severidad de la Adicción - versión europea).-

Se trata de la adaptación europea de la $5^{\text {a }}$ versión del Addiction Severity Index -ASI- (39-40), Ilevada a cabo dentro del marco de la Comunidad Económica Europea a través del grupo de expertos COST-A6 ${ }^{(41)}$ 
El Índice de Severidad de la Adicción (ASI / EuropASI) es un instrumento basado en un formato de entrevista clínica semiestructurada cuyo propósito es la evaluación multidimensional de los problemas relacionados con el consumo de sustancias psicoactivas. Proporciona una valoración de la gravedad, entendida como 'necesidad de tratamiento adicional', de cada uno de los problemas comúnmente relacionados con el consumo de drogas. Explora siete áreas problemáticas a través de siete escalas, a saber: el 'estado médico general', el 'empleo / apoyos', el 'consumo de drogas' el 'consumo de alcohol', la 'situación legal', las 'relaciones socio-familiares' y el 'estado psicopatológico'. Las puntuaciones totales de cada una de las secciones siguen un modelo lineal; un valor más alto se interpreta como un problema más grave, más concretamente, como 'una mayor necesidad de tratamiento adicional' (42).

La gravedad de cada una de las dimensiones medidas por el EuropASI se puntúa de forma individual e independiente a través de la combinación de ítems objetivos y subjetivos ubicados en cada escala. De este modo, para cada dimensión es posible calcular una puntuación VGE ('Valoración de la Gravedad por el Entrevistador') que deriva de una valoración clínica global que hace el entrevistador sobre la gravedad o nivel de deterioro del paciente en un área determinada y que es evaluado a través de una escala con rango de 0 a 9. Para la determinación de esta puntuación VGE, el entrevistador ha de seguir un procedimiento sistemático y estandarizado. A este respecto, la versión del EuropASI y la Guía-Manual de Entrenamiento y Administración de la misma utilizados en nuestro estudio corresponden con la adaptación coordinada por el grupo de Bobes ${ }^{(43)}$.

Por otra parte, se ha considerado para nuestro estudio la valoración subjetiva proporcionada por el paciente. En cada sección se realizan dos preguntas que tratan de valorar la gravedad del problema percibida por el entrevistado: '¿cuánto le han preocupado o molestado, en los últimos 30 días, estos problemas ... que ha tenido?', y '¿qué importancia tiene, ahora, para Ud. recibir ayuda o tratamiento para estos problemas ...?'. En ambos casos se utiliza una escala de valoración del paciente de cinco puntos con rango de 0 a 4 . De entre estos dos ítems subjetivos, se ha tomado al primero de ellos como criterio de la Valoración de la Gravedad por el Paciente (Puntuación VGP), y se han transformado sus puntuaciones para cada una de las dimensiones en una escala decimal para permitir su comparación con las puntuaciones VGE, las proporcionadas por el entrevistador.

\section{Análisis estadísticos}

Para la descripción de la muestra y la valoración de las puntuaciones del EuropASI y FAP se han calculado el recuento de frecuencias (n) y porcentajes (\%) en el caso de variables cualitativas, y la media (M) y desviación estándar (DE) para las variables cuantitativas. Las puntuaciones VGE y VGP han sido comparadas entre sí a partir una prueba t-test (t de student) para muestras relacionadas. La asociación entre las dimensiones que componen el EuropASI, el FAP, respecto a sí mismas, o las asociaciones entre las escalas de ambos instrumentos fueron estimadas a partir del cálculo de los coeficientes de correlación producto-momento de Pearson ( $r$ ).

Para interpretar los resultados se ha elegido un nivel de significación de $p<0,05$, para un intervalo de confianza del 95\% (IC 95\%) en el supuesto de hipótesis bidireccional. En general, estos análisis de datos han de considerarse como exploratorios. Todos los análisis se han realizado mediante el programa SPSS V.10 ${ }^{\left({ }^{44}\right)}$.

Figura 1.- Puntuaciones de la Valoración de la Gravedad por el Entrevistador (VGE) y Valoración de la Gravedad por el Paciente (VGP) en las áreas del EuropASI.

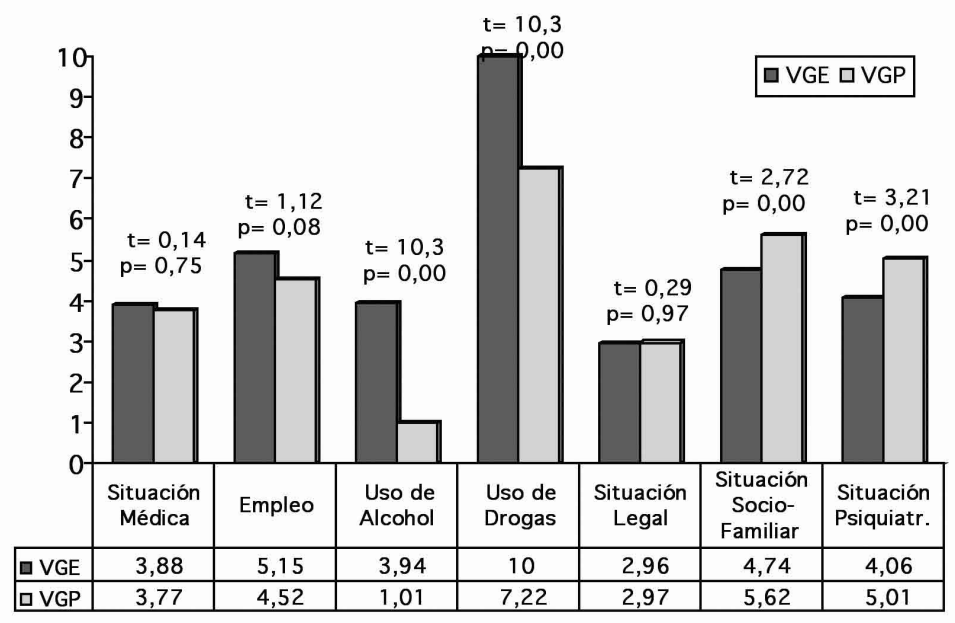




\section{RESULTADOS}

En la Figura 1 se muestran las puntuaciones de la Valoración de la Gravedad por el Entrevistador (VGE) y la Valoración de la Gravedad por el Paciente (VGP), y las pruebas de contraste de diferencias entre ambas puntuaciones para cada una de las áreas del EuropASI. Las áreas más comprometidas (puntuaciones cercanas a 5 ó superiores), tanto para el caso de las VGE como para las VGP, fueron el uso de drogas, el empleo y las situaciones socio-familiar y psiquiátrica, entre las que destaca con valoración de mayor gravedad el uso de drogas. Se han encontrado diferencias estadísticamente significativas entre las valoraciones de gravedad ofrecidas por el entrevistador y el paciente en cuatro de las áreas: uso de alcohol, uso de drogas, situación sociofamiliar y situación psiquiátrica; las dos primeras con mayor gravedad percibida por parte del entrevistador, y en las dos últimas por parte del paciente.

La concordancia entre VGE y VGP, estimada a través del coeficiente de correlación de Pearson, fue estadísticamente significativo $(p<0,05)$ en cuatro de las áreas del EuropASI (Situación médica, uso de alcohol, y áreas legal, socio-familiar y psiquiátrica con valores $r$ de 0,$38 ; 0,41 ; 0,62 ; 0,17$ y 0,43 , respectivamente), no así en el área de empleo $(r=0,14 ; p=0,085)$. La correlación entre las diferentes áreas de la VGE y VGP respecto al uso de drogas no pudo calcularse por falta de variabilidad en la puntuación VGE en esta área.

En la Tabla 1 se presentan los índices de correlación de Pearson entre las puntuaciones VGP (matriz triangular inferior) y las VGE (matriz triangular superior) de las subescalas del EuropASI. Del conjunto de asociaciones posibles entre las VGP de las 7 áreas del EuropASI, tan solo cinco han resultado estadísticamente significativas (Tabla 1, matriz inferior) mostran- do una correlación positiva. Cuatro de ellas se hallan entre el área de empleo/apoyos y las áreas siguientes: situación médica $(r=0,30)$, situación legal $(r=0,41)$, situación socio-familiar $(r=0,24)$ y situación psiquiátrica $(r=0,21)$; la quinta asociación se encuentra entre la situación psiquiátrica y la socio-familiar $(r=0,47)$.

Respecto a las asociaciones entre las puntuaciones VGE (Tabla 1, matriz superior), la severidad valorada por el evaluador respecto a la situación familiar del paciente está significativamente asociada a la gravedad percibida sobre la situación social $(r=0,44)$ y psiquiátrica $(r=0,41)$, con la gravedad por el uso de drogas $(r=0,36)$ y con la percibida respecto a la situación médica $(r=0,28)$. También se encuentran correlaciones significativas entre la gravedad percibida por la situación psiquiátrica y la gravedad respecto al uso de drogas $(r=0,43)$, la situación médica $(r=0,32)$, la situación social $(r=0,32)$ y la preocupación por el empleo/ apoyos $(r=0,29)$ que manifiesta el paciente.

En la Tabla 2 se presentan los valores descriptivos e índices de correlación de Pearson entre las puntuaciones que componen el FAP. Las dimensiones de Satisfacción y Recursos familiares obtienen una puntuación media de 5 sobre un máximo de 10; las otras dos dimensiones ofrecen puntuaciones que oscilan entre 3,45 y 4,77 , indicativos de un bajo nivel de estrés familiar y comunicación, respectivamente. Respecto a las asociaciones entre las subescalas del FAP se observan correlaciones estadísticamente significativas entre todas ellas, diferenciándose una asociación negativa entre el estrés y las escalas de comunicación, satisfacción y recursos familiares, y correlaciones positivas entre estas tres últimas.

En la Tabla 3 se presentan los coeficientes de correlación de Pearson entre las puntuaciones de las escalas del FAP y las Puntuaciones de Valoración de Gravedad (VGE y VGP) de las áreas del EuropASI. La

\section{Tabla 1.- Índices de correlación de Pearson entre las Puntuaciones de la Valoración de Gravedad del Entrevistador -VGE- (matriz triangular superior) y la Valoración de la Gravedad del Paciente -VGP- (matriz triangular inferior) de las subescalas del EuropASI $(n=140)$}

\begin{tabular}{lcccccccc|}
\hline Áreas & & SM & ES & UA & UD & SL & SSF & SP \\
\hline Situación Médica & SM & & $-0,012$ & 0,058 & 0,019 & $-0,026$ & $0,279^{* *}$ & $0,321^{* *}$ \\
Empleo / Apoyo & EA & $0,300^{* *}$ & & 0,161 & 0,085 & 0,113 & $0,195^{*}$ & $0,295^{* *}$ \\
Uso de Alcohol & UA & $-0,134$ & $-0,132$ & & 0,068 & $-0,007$ & $0,170^{* *}$ & 0,124 \\
Uso de Drogas & UD & $\varnothing$ & $\varnothing$ & $\varnothing$ & & $-0,042$ & $0,364^{* *}$ & $0,435^{* *}$ \\
Situación Legal & SL & 0,125 & $0,415^{* *}$ & 0,081 & $\varnothing$ & & $-0,071$ & $-0,060$ \\
Situación Socio-Familiar & SSF & 0,151 & $0,245^{* *}$ & 0,075 & $\varnothing$ & 0,052 & & $0,437^{* *}$ \\
Situación Psiquiátrica & SP & 0,154 & $0,214^{*}$ & 0,144 & $\varnothing$ & 0,049 & $0,471^{* *}$ & \\
\hline
\end{tabular}

$\varnothing$ - No es posible calcular la correlación por falta de variabilidad en la puntuación VGE del área uso de drogas * $p<0,05 ; * * p<0,01$ 
Tabla 2.- Valores descriptivos e índices de correlación de Pearson entre las puntuaciones del FAP $(n=140)$

\begin{tabular}{|lccccccc|}
\hline Dimensiones del FAP & E & C & S & RF & Media & (D.T.) \\
\hline Estrés & E & 1,00 & & & & 3,45 & $(1,40)$ \\
Comunicación & C & $-0,453^{* *}$ & 1,00 & & & 4,77 & $(2,38)$ \\
Satisfacción & S & $-0,541^{* *}$ & $0,801^{* *}$ & 1,00 & & 5,03 & $(2,34)$ \\
Recursos Familiares & RF & $-0,474^{* *}$ & $0,824^{* *}$ & $0,798^{* *}$ & 1,00 & 5,00 & $(2,06)$ \\
\hline${ }^{*} p<0,05 ;{ }^{* *} p<0,01$ & & & & & & & \\
\hline
\end{tabular}

Tabla 3.- Asociación entre las puntuaciones de severidad (PSE) de las subescalas del EuropASI y las puntuaciones del FAP-FACES en la línea base $(n=140)$

\begin{tabular}{|lcccccccc|}
\hline \multirow{2}{*}{$\begin{array}{l}\text { Dimensiones del FAP } \\
\text { Áreas del EuropASI }\end{array}$} & \multicolumn{2}{c}{ Estrés } & \multicolumn{2}{c}{ Comunicación } & \multicolumn{2}{c|}{ Satisfacción } & \multicolumn{2}{c|}{ Rec. Familiares } \\
\cline { 2 - 9 } & VGE & VGP & VGE & VGP & VGE & VGP & VGE & VGP \\
\hline Situación Médica & 0,019 & $0,225^{*}$ & $-0,054$ & $-0,088$ & $-0,027$ & $-0,137$ & $-0,061$ & $-0,105$ \\
Empleo / Apoyo & 0,152 & 0,139 & $-0,056$ & $-0,203^{*}$ & $-0,070$ & $-0,219^{* *}$ & $-0,139$ & $-0,217^{* *}$ \\
Uso de Alcohol & $-0,029$ & 0,050 & $-0,056$ & $-0,077$ & $-0,041$ & $-0,063$ & $-0,030$ & $-0,098$ \\
Uso de Drogas & $\varnothing$ & $0,260^{* *}$ & $\varnothing$ & $-0,180^{*}$ & $\varnothing$ & $-0,263^{* *}$ & $\varnothing$ & $-0,209^{*}$ \\
Situación Legal & 0,005 & 0,022 & 0,008 & $-0,048$ & $-0,049$ & $-0,095$ & $-0,002$ & $-0,002$ \\
Situación Socio-Familiar & $0,323^{* *}$ & $0,339^{* *}$ & $-0,371^{* *}$ & $-0,253^{* *}$ & $-0,378^{* *}$ & $-0,247^{* *}$ & $-0,387^{* *}$ & $-0,239^{* *}$ \\
Situación Psiquiátrica & $0,275^{* *}$ & $0,265^{* *}$ & $-0,346^{* *}$ & $-0,229^{* *}$ & $-0,367^{* *}$ & $-0,253^{* *}$ & $-0,412^{* *}$ & $-0,266^{* *}$ \\
\hline
\end{tabular}

$\varnothing$ - No es posible calcular la correlación por falta de variabilidad en la puntuación VGE del área uso de drogas * $p<0,05 ; * * p<0,01$

severidad valorada por el clínico (VGE) tan solo se asocia con el FAP en dos áreas, la situación socio-familiar y la psiquiátrica; de forma que una alta puntuación de severidad en estas áreas se relacionaría con un mayor estrés y con menores niveles de comunicación, satisfacción y recursos familiares. Para el resto de áreas del EuropASI, la valoración de la severidad en los problemas relacionados con las mismas no presenta relaciones significativas con las facetas de clima familiar exploradas.

Atendiendo a la gravedad valorada por el paciente (VGP), la asociación entre las áreas uso de drogas, situación familiar, situación psiquiátrica y empleo respecto a las escalas de clima familiar del FAP siguen el mismo perfil: correlaciones positivas con el estrés y negativas con la comunicación, satisfacción y recursos familiares. Es decir, una mayor percepción como problema por parte del usuario en estas áreas del EuropASI se relacionaría con un mayor nivel de estrés y con una peor comunicación, satisfacción y nivel de recursos familiares. Por otra parte, también se encuentra una correlación positiva entre la situación médica y el nivel de estrés (a medida que aumenta la preocupación del sujeto por su situación médica aumenta tam- bién el nivel de estrés). A destacar que no se hallan asociaciones estadísticamente significativas entre la valoración como problema por parte del usuario en las áreas de uso de alcohol y situación legal, y las escalas de clima familiar.

\section{DISCUSIÓN}

El objetivo de este estudio ha sido comprobar la relación entre el funcionamiento familiar y la severidad de la toxicomanía evaluada tanto por el paciente como por el entrevistador en una muestra de pacientes toxicómanos en tratamiento. El análisis de resultados permite algunos comentarios de interés tanto en lo que atañe a la valoración de severidad, como al funcionamiento familiar, como a la interacción de estos dos factores.

Respecto a la valoración de severidad de los problemas vinculados a la adicción se observa una apreciación diferencial entre el juicio que realiza el paciente 
y el evaluador en algunas de las dimensiones exploradas. La situación médica, legal y el empleo son valorados de forma equivalente y en general con puntuaciones inferiores a 5 , indicativas de una situación de moderada-baja severidad. Las diferencias se encuentran fundamentalmente en la valoración del consumo de sustancias y/o alcohol, donde el evaluador atribuye una mayor severidad, y la valoración de la situación psiquiátrica y socio-familiar, donde es el paciente quien expresa una mayor severidad en relación al juicio del clínico. Estas diferencias significativas en la apreciación de la severidad por las partes interesadas podrían estar motivadas por las diferentes expectativas sobre el tratamiento. A este respecto, varias hipótesis podrían plantearse. Por una parte, el clínico asume que el paciente acude para buscar una solución a su problema de adicción, por lo que la severidad centrada en esta área pudiera estar sobrevalorada. Esta hipótesis no es del todo plausible ya que si bien la valoración de severidad que hace el evaluador del consumo de drogas es de 10, la que realiza sobre el uso de alcohol se sitúa en una puntuación de 3,9. De producirse el sesgo hubiera de apreciarse en ambos consumos, si bien es cierto que el motivo de demanda de tratamiento es por adicción a drogas no alcohólicas. Resultados similares se han encontrado en otros estudios ${ }^{(45-48)}$. Otra posible hipótesis es que los pacientes infravaloran la gravedad de su consumo respecto al criterio de los evaluadores, aunque hay que observar que la puntuación de severidad es de 7,22, una puntuación que ha de considerarse alta. Desde nuestro punto de vista no se trataría tanto de una falta de conciencia de enfermedad, en tanto en cuanto juzgan su situación psiquiátrica y sociofamiliar con mayor severidad que los clínicos. Esta discrepancia en los juicios sobre la salud de pacientes y clínicos sobre la situación de los primeros, ahonda en un problema largamente discutido: la necesidad de considerar las apreciaciones subjetivas de los implicados en los procesos clínicos y la conveniencia de contrastarlos con datos más objetivos.

Del mismo modo que se produce una valoración diferencial en la percepción del grado de severidad, la forma en que las áreas exploradas se ven asociadas varía entre el evaluador y el evaluado. En el caso del paciente, el área de empleo/apoyo se asocia positivamente con cuatro de las áreas, y la situación psiquiátrica con la sociofamiliar. Es decir, para el paciente la severidad percibida en su situación de empleo está significativamente asociada a una mayor percepción de severidad con su situación médica, psiquiátrica, legal y sociofamiliar, y esta última con su situación psiquiátrica. Las relaciones son bidireccionales, por lo que no podemos establecer una conexión causal de un área sobre las otras. Lo que resulta de interés es que no se ha encontrado ninguna asociación entre la severidad por el uso de drogas y/o alcohol con el resto de áreas que supuestamente se verían alteradas en el caso de un problema de adicción. Desde esta perspectiva, para el paciente, tener problemas de tipo psiquiátrico o con la familia no se asocia con consumir drogas, lo cual no deja de ser paradójico.

Otras valoraciones podemos observar en el caso de las asociaciones entre las puntuaciones de severidad ofrecidas por el evaluador. En este caso, son las áreas sociofamiliar y psiquiátrica las que principalmente se asocian con las demás, y de forma especial con el consumo de drogas. Este tipo de hallazgo es más plausible respecto a las hipótesis subyacentes al uso del ASI/EuropASI, donde la severidad de los problemas asociados al consumo de drogas tendría una expresión en un mayor número de áreas. Lo interesante en este caso, es que las áreas menos clínicas, como son el empleo o la situación legal, no hallan una relación de su grado de severidad con la severidad manifestada en otras áreas.

Respecto a la situación familiar, el análisis de las dimensiones del FAP muestra, en parte, resultados en consonancia con lo esperado: se encuentran asociaciones positivas entre la comunicación, la satisfacción y los recursos, y negativas entre éstas y el estrés familiar. Es decir, por ejemplo, un mayor grado de comunicación en la familia se asocia a una mayor satisfacción, mientras que un mayor nivel de estrés implicaría una menor comunicación o un nivel de satisfacción más deficitario. Resultados análogos han sido observados en diferentes estudios nacionales e internacionales ${ }^{(3,5,49-50)}$. Lo que destaca de nuestros resultados es el bajo nivel de estrés familiar percibido por los pacientes que inician tratamiento. En consonancia con otros hallazgos ${ }^{(5,51-54)}$, esperábamos que el grado de estrés fuera mayor, en tanto que la evidencia ha mostrado que las situaciones de consumo de drogas por un miembro familiar, y sobre todo cuando este consumo es conocido por sus componentes, es un factor de estrés familiar de alto grado ${ }^{(53,55)}$. Una posible explicación al efecto hallado podría encontrarse en la relajación que los pacientes, y quizá también la familia, experimentan al iniciarse un proceso terapéutico que reduce la tensión familiar ante la expectativa de una solución para el problema de adicción. Nótese que las puntuaciones medias en las otras tres dimensiones del FAP, propias de un buen funcionamiento familiar, son moderadas quizá también expresión de esa acomodación que hemos propuesto. Una posible verificación de la hipótesis subyacente a nuestra propuesta consistiría en examinar estos indicadores de funcionamiento familiar durante un proceso de recaída, donde el estrés volvería a presentarse y las otras dimensiones se verían también alteradas. Tal verificación supone un proceso de seguimiento basado en un diseño longitudinal, que será objeto de futuras aproximaciones por parte de nuestro grupo. 
En definitiva, nuestro estudio aporta resultados que muestran la relación entre la severidad de la adicción y el funcionamiento familiar. Queda pendiente observar si el proceso terapéutico tendrá influencia en la forma de relación entre estos factores al cabo del tiempo. Está será la línea de investigación a desarrollar en próximos proyectos.

\section{REFERENCIAS}

1. Vilaregut A, Pérez C, Sánchez-Carbonell J, Alós C. La dinámica familiar con un hijo drogodependiente. Itaca 2000; 5: 31-56

2. Dakof A. Understanding gender differences in adolescent drug abuse: Issues of comorbidity and family functioning. J Psychoactive Drugs 2000; 32: 25-32.

3. Charvoz L, Bodenmann G, Hermann E. The importance of family variables for the drug consumption of adolescents. Kindheit und Entwicklung 2002; 11: 14-20.

4. McArdle P, Wiegersma A, Gilvarry E, Kolte B, McCarthy S, Fitzgerald M, Brinkley A, Blom M, Stoeckel I, Pierolini A, Michels I, Johnson R, Quensel S. European adolescent substance use: the roles of family structure, function and gender. Addiction 2002; 97(3): 329-36.

5. Coviello DM, Alterman Al, Cacciola JS, Rutherford MJ, Zanis DA. The role of family history in addiction severity and treatment response. J Subst Abuse Treat 2004; 26(1): 303-13.

6. Kumpfer KL, Alvarado R, Whiteside HO. Family-based interventions for substance use and misuse prevention. Subst Use Misuse 2003; 38(11-13): 1759-87.

7. Szapocznik J, Hervis O, Schwartz S. Brief Strategic Family Therapy for Adolescent Drug Abuse. NIDA, Therapy Manuals for Drug Addiction Col. Bethesda, Maryland, 2003.

8. Herman MA, McHale SM. Coping with parental negativity: Links with parental warmth and child adjustment. J Appl Develop Psychol 1993; 14: 121-36.

9. Johnson V, Pandina RJ. Effects of the family environment on adolescent substance use, delinquency, and coping styles. Amer J Drug Alc Abuse 1991; 17: 71-88.

10. Otero JM, Mirón L, Luengo A. Influence of family and peer group on the use of drugs by adolescents. Int $J$ Addict 1989; 24: 1065-1082.

11. Bragado C, Bersabé R, Carrasco, F. Factores de riesgo para los trastornos conductuales, de ansiedad, depresivos y de eliminación en niños y adolescentes. Psicothema 1999; 11: 939-956.

12. Castells P. Relaciones familiares. Barcelona: STJ; 1999.

13. Franco GE. La comunicación en familia. Madrid: Palabra; 1994.

14. Lyons EM. A comparison of family patterns among Hispanic adolescent drug-using and non-drug-using clinical populations. Dissertation Abstracts International
Section A: Humanities and Social Sciences 1996; 56 : 4663.

15. Spooner C. Causes and correlates of adolescent drug abuse and implications for treatment. Drug Alc Review 1999; 18: 453-475.

16. Elzo J. Drogas y Escuela V. Vitoria: Servicio de Publicaciones del Gobierno Vasco; 1996.

17. Pardeck JT. A multiple regression analysis of family factors affecting the potential for alcoholism in college students. Fam Ther 1991; 15: 118-121.

18. Pons J. Autoconcepto, comunicación familiar y consumo de drogas en alumnos de Segundo Ciclo de E.G.B. Valencia: Universitat de Valencia; 1989.

19. Protinsky H, Shilts L. Adolescent substance use and family cohesion. Fam Ther 1990; 17: 173-5.

20. Vielva I, Pantoja L, Abeijón JA. El funcionamiento de la familia con hijos (consumidores y no consumidores) de comportamientos no problemáticos. Bilbao: Universidad de Deusto; 2001.

21. Lavee $\mathrm{Y}, \mathrm{McC}$ cubbin $\mathrm{HI}$, Olson DH. The effect of stressful life events and transitions on family functioning and well-being. J Marriage Fam 1987; 49: 857-873.

22. McCubbin HI, Needle RH, Wilson M. Adolescent health risk behaviours: Family stress and adolescent coping as critical factors. Special issue: The family and health care. Fam Relat 1985; 34: 51-62.

23. Malkus BM. Family dynamic and structural correlates of adolescent substance abuse: A comparison of families of non-substance abusers and substance abusers. J Child Adolesc Subst Abuse 1994; 3: 39-52.

24. Carvalho V, Pinsky I, de Souza e Silva R, Carlini-Cotrim B. Drug and alcohol use and family characteristics: A study among Brazilian high-school students. Addiction 1995; 90: 65-72.

25. Fergusson D, Horwood L, Lynskey M. Parental separation, adolescent psychopathology, and problem behaviours. J Am Acad Child Adolesc Psychiatry 1994; 33: 1122-1131.

26. Velleman R. Intergenerational effects. A review of environmentally oriented studies concerning the relationship between parental alcohol problems and family disharmony in the genesis of alcohol and other problems II: The intergenerational effects of family disharmony. Int J Addict 1992; 27: 367-389.

27. McQueen A, Getz JG, Bray JH. Acculturation, substance use, and deviant behavior: examining separation and family conflict as mediators. Child Dev 2003; 74(6): 1737-50.

28. Lewis RA. The family and addictions: An introduction. Fam Relat 1989; 38: 254-257.

29. Vecino C. Características estructurales y dinámicas de familias con un hijo heroinómano: Estudio caso control de una población de un sector de Barcelona desde un enfoque sistémico relacional. [Tesis doctoral no publicada]. Barcelona: Universitat Autònoma de Barcelona; 1990. 
30. Muñoz-Rivas MJ, Graña JL. Factores familiares de riesgo y de protección para el consumo de drogas en adolescentes. Psicothema 2001; 13: 87-94.

31. American Psychiatric Association. Diagnostic and statistical manual of mental disorders: DSM-IV. Washington: American Psychiatric Association; 1997.

32. Olson DH, Tiese, JW, Gorall DM, Fitterer C. Family Assessment Package. Unpublished Manuscript, University Of Minnesota, St. Paul; 1996.

33. Sanz M, Iraurgi I, Martínez-Pampliega A. Evaluación del funcionamiento familiar en toxicomanías: Adaptación española y características de adecuación métrica del FAP-FACES IV. En: Iraurgi I, González-Saiz F. (Eds.). Instrumentos de Evaluación en drogodependencias. Madrid: Aula Médica; 2002. p. 403-434.

34. Olson DH, Wilson M. Family Satisfaction Scale. En Olson DH, Mccubbin H, Barnes H, Larsen A, Muxen M, Wilson M. (Eds). Family Inventories Manual. St. Paul: University Of Minnesota; 1983. p. 92-110.

35. McCubbin HI, Patterson JM, Wilson L. Family Inventory of Life Events and Changes (FILE), form A. St. Paul, MN: University of Minnesota; 1981.

36. Barnes $\mathrm{HL}$, Olson DH. Parent-adolescent communication scale. En: Olson DH, McCubbin HI, Barnes H, Larsen A, Muxen M, Wilson M. (Eds.). Family inventories: Inventories used in a national survey of families across the family life cycle. St. Paul, MN: University of Minnesota; 1982: pp. 51-63.

37. Olson DH, Stewart KL, Wilson LR. Health and stress profile (HSP), revised. Minneapolis: Profile of Health Systems; 1990.

38. Olson DH, Larsen A, McCubbin HI. Family Strengths. En: Olson DH, McCubbin HI, Barnes H, Larsen A, Muxen M, Wilson M. (Eds.). Family inventories: Inventories used in a national survey of families across the family life cycle. St. Paul, MN: University of Minnesota; 1982. p. 78-92.

39. McLellan AT, Kushner H, Metzger D, Peters R, Smith I, Grissom G, Pettinati H, Argeriou M. The fifth edition of the Addiction Severity Index. J Subst Abuse Treat 1992; 9: 199-213

40. McLellan AT, Luborsky L, O’Brien CP, Woody GE. An improved diagnostic evaluation instrument for substance abuse patients. The Addiction Severity Index. J Nerv Ment Dis 1980; 168: 26-33.

41. Kokkevi A, Hartgers C. EuropASI: European adaptation of a multidimensional assessment instrument for drug and alcohol dependence. Eur Addict Res 1995; 1(4): 208-10.

42. González-Saiz F, Iraurgi I, Fernández-Gómez C, MartínezDelgado JM, Marchena JC. Instrumentos de evaluación multidimensional en los trastornos relacionados con sustancias. En: XIII Congreso Nacional Sociedad Española de Toxicomanías. Libro de Actas. Cádiz: Sociedad Española de Toxicomanías; 2001. p. 39-97.
43. Bobes J, González MP, Sáiz PA, Bousoño M. Índice europeo de severidad de la adicción: EuropASI. Versión española. Actas de la IV Reunión Interregional de Psiquiatría 1996; 201-218.

44. Norusis MJ. SPSS 10. A Guide to data analysis. Book News, SPSS Inc., Portland; 2000.

45. Martin C, Grabot D, Auriacombe M, Brisseau S, Daulouede JP, Tignol J. Descriptive studies of the use of the Addiction Severity Index in France. Encephale 1996; 22(5): 359-363.

46. Fernández-Miranda JJ, González-Portilla MP, Sáiz-Martínez PA, Gutiérrez-Cienfuegos E, Bobes J. Calidad de vida y severidad de la adicción en heroinómanos en mantenimiento prolongado con metadona. Adicciones 1999; 11(1): 43-52.

47. Scheurich A, Muller MJ, Wetzel H, Anghelescu I, Klawe C, Ruppe A, Lorch B, Himmerich H, Heidenreich M, Schmid G, Hautzinger M, Szegedi A. Reliability and validity of the German version of the European Addiction Severity Index (EuropASI). J Stud Alc 2000; 61(6): 916919.

48. González-Saiz F, Salvador L, Martínez-Delgado JM, López-Cárdenas A, Ruz I, Guerra D. El Addiction Severity Index (ASI): A propósito de una revisión. En: Iraurgi I, González-Saiz F. (Eds.). Instrumentos de Evaluación en drogodependencias. Madrid: Aula Médica; 2002, pp. 235-269.

49. Wampler R, Fischer J, Thomas M, Lyness K. Young adult offspring and their families of origin: cohesion, adaptability, and addiction. J Subst Abuse 1993; 5(2): 195201.

50. Pickens RW, Preston KL, Miles DR, Gupman AE, Jonson EO, Newlin DB, Soriano J, van den Bree MB, Umbricht A. Family history influence on drug abuse severity and treatment outcome. Drug Alcohol Depend 2001; 61(3): 261-270.

51. Costantini MF, Wermuth L, Sorensen JL, Lyons JS. Family functioning as a predictor of progress in substance abuse treatment. J Subst Abuse Treat 1992; 9(4): 331335.

52. Stewart MA, Brown SA. Family functioning following adolescent substance abuse treatment. J Subst Abuse 1993; 5(4): 327-339.

53. Killeen T, Brady KT. Parental stress and child behavioral outcomes following substance abuse residential treatment. Follow-up at 6 and 12 months. J Subst Abuse Treat 2000; 19(1): 23-29.

54. Latimer WW, Newcomb M, Winters KC, Stinchfield RD. Adolescent substance abuse treatment outcome: the role of substance abuse problem severity, psychosocial, and treatment factors. J Consult Clin Psychol 2000; 68(4): 684-696.

55. Olsson CA, Coffey C, Toumbourou JW, Bond L, Thomas $L$, Patton G. Family risk factors for cannabis use: a population-based survey of Australian secondary school students. Drug Alcohol Rev 2003; 22(2): 143-52. 


\title{
ANEXO \\ FAP (Family Assessment Package)
}

Olson DH, Tiesel JW, Gorall DM, Fitterer C. Family Assessment Package. Unpublished Manuscript, University Of Minnesota, St. Paul, 1996.

Adaptado por: Sanz M, Iraurgi I, Martínez-Pampliega. Evaluación del funcionamiento familiar en toxicomanías: Adaptación española y características de adecuación métrica del FAP-FACES IV. En Iraurgi I, González-Saiz F (Eds.). Instrumentos de evaluación en drogodependencias. Madrid, Aula Médica; 2002; 403-434.

A continuación van a aparecer unas frases en las que se hace referencia a la familia más cercana, con la que tú convives. Se pueden incluir también otros miembros de la familia que no viven en tu misma casa, pero que sí se relacionan con vosotros de un modo importante. De las opciones dadas, elige aquella que mejor describa tu situación familiar actual. Redondea el número, del uno al cinco, que describa la respuesta que hayas elegido.

\author{
Escala de Estrés Familiar \\ Durante el último año, ¿con qué frecuencia estas situaciones crearon tensión en tu familia? \\ 1)Nunca; 2) En muy pocas ocasiones; 3) A veces; 4) Con Frecuencia; 5) Muy Frecuentemente \\ Discusiones entre padre/s e hijo/s \\ $\begin{array}{lllll}1 & 2 & 3 & 4 & 5\end{array}$ \\ Viajes de negocios de los padres \\ $\begin{array}{lllll}1 & 2 & 3 & 4 & 5\end{array}$ \\ Mucho gasto, por encima de las posibilidades, por medio de tarjetas de crédito \\ $\begin{array}{lllll}1 & 2 & 3 & 4 & 5\end{array}$ \\ Enfermedades físicas o muerte de un miembro de la familia \\ $1 \quad 2 \quad 3 \quad 4 \quad 5$ \\ La escasez de participación de los hijos en las labores de casa \\ $\begin{array}{lllll}1 & 2 & 3 & 4 & 5\end{array}$ \\ Los conflictos se quedan sin resolver \\ $\begin{array}{lllll}1 & 2 & 3 & 4 & 5\end{array}$ \\ Dificultad al pagar las cuentas mensuales \\ Dificultad de los padres en el cuidad de los hijos/as \\ Problemas afectivos con los miembros de la familia (discusiones, etc.) \\ Suspensos en relación con el trabajo escolar de los hijos/as \\ Cuestiones 0 asuntos con las familias del padre 0 de la madre \\ $\begin{array}{lllll}1 & 2 & 3 & 4 & 5\end{array}$ \\ $\begin{array}{lllll}1 & 2 & 3 & 4 & 5\end{array}$ \\ $\begin{array}{lllll}1 & 2 & 3 & 4 & 5\end{array}$ \\ $\begin{array}{lllll}1 & 2 & 3 & 4 & 5\end{array}$ \\ $\begin{array}{lllll}1 & 2 & 3 & 4 & 5\end{array}$ \\ $\begin{array}{lllll}1 & 2 & 3 & 4 & 5\end{array}$ \\ Las tareas de la casa se quedan sin hacer \\ El/los hijo/s tienen responsabilidades por encima o por debajo de los que corresponde a su edad \\ $\begin{array}{lllll}1 & 2 & 3 & 4 & 5\end{array}$ \\ Preocupaciones a causa del consumo de drogas \\ Dificultad de los padres para controlar a los hijos/as (disciplina) \\ $\begin{array}{lllll}1 & 2 & 3 & 4 & 5\end{array}$ \\ $\begin{array}{lllll}1 & 2 & 3 & 4 & 5\end{array}$ \\ $\begin{array}{lllll}1 & 2 & 3 & 4 & 5\end{array}$ \\ Problemas con respecto al reparto de las tareas de la casa \\ Un embarazo o reciente nacimiento de un hijoFalta de tiempo para relajarse o desconectar \\ Problemas o dificultades de adaptación por el cambio de domicilio \\ $\begin{array}{lllll}1 & 2 & 3 & 4 & 5\end{array}$ \\ $\begin{array}{lllll}1 & 2 & 3 & 4 & 5\end{array}$ \\ Obligaciones familiares

\section{Escala de Recursos Familiares}

Indica en qué medida, cada una de las siguientes frases describe a tu familia

1) No describe nada a mi familia; 2) Sólo le describe ligeramente; 3) Describe a veces a mi familia; 4) En general, sí describe a mi familia; 5) Describe muy bien a mi familia

Podemos expresar nuestros sentimientos

Nos sentimos capaces de afrontar los problemas cuando surgen

Tenemos confianza en nosotros mismos

Tenemos confianza en que podemos resolver los problemas eficazmente

Los miembros de la familia somos leales a la familia

Conseguir nuestros objetivos nos resulta fácil

Los miembros de la familia decimos lo que pensamos de los otros de forma positiva

Compartimos valores y creencias muy similares

Creemos que las cosas nos van bien en nuestra familia

Los miembros de la familia nos respetamos entre nosotros

Pocos problemas quedan por resolver en nuestra familia 


\section{Escala de Comunicación Familiar}

1) No describe nada a mi familia; 2) Sólo le describe ligeramente; 3) Describe a veces a mi familia; 4) En general, sí describe a mi familia; 5) Describe muy bien a mi familia

Los miembros de mi familia estamos satisfechos con la forma de comunicarnos

Los miembros de mi familia sabemos escuchar

Los miembros de la familia nos expresamos afecto entre nosotros

En nuestra familia compartimos los sentimientos abiertamente

Disfrutamos pasando tiempo juntos

Los miembros de la familia discutimos los sentimientos e ideas entre nosotros

Cuando los miembros de la familia preguntamos algo, las respuestas son sinceras

Los miembros de la familia intentamos comprender los sentimientos de los otros

Los miembros de la familia resolvemos tranquilamente los problemas

En nuestra familia expresamos nuestros verdaderos sentimientos

\section{Escala de Satisfacción Familiar}

Por favor, indica tu grado de satisfacción con lo siguiente:

1) Muy insatisfecho ; 2) Insatisfecho; 3) Ni satisfecho, ni insatisfecho; 4) Satisfecho; 5) Muy satisfecho

El grado de cercanía entre los miembros de la familia

La capacidad de tu familia para afrontar las situaciones de tensión

La capacidad de tu familia para ser flexible 0 adaptable

La capacidad de tu familia para compartir experiencias positivas

La calidad de la comunicación entre los miembros de la familia

La capacidad de tu familia para resolver conflictos

La cantidad de tiempo que pasáis juntos como una familia

El modo en que los problemas son tratados

Lo justa que es la crítica en tu familia

$\begin{array}{lllll}1 & 2 & 3 & 4 & 5\end{array}$

La preocupación de cada uno de nosotros por los otros miembros de la familia 
